\title{
Right ventricular peak systolic longitudinal strain is a sensitive marker for right ventricular deterioration in adult patients with tetralogy of Fallot
}

\author{
Roderick W. C. Scherptong $\cdot$ Sjoerd A. Mollema $\cdot$ Nico A. Blom • \\ Lucia J. M. Kroft · Albert de Roos · Hubert W. Vliegen • \\ Ernst E. van der Wall · Jeroen J. Bax · Eduard R. Holman
}

Received: 16 January 2009/ Accepted: 9 July 2009/Published online: 30 July 2009

(C) The Author(s) 2009. This article is published with open access at Springerlink.com

\begin{abstract}
The aim of this study was to evaluate the feasibility of right ventricular (RV) longitudinal peak systolic strain (LPSS) assessment for the follow-up of adult patients with corrected tetralogy of Fallot (TOF). Adult patients $(n=18)$ with corrected TOF underwent echocardiography and CMR twice with a time interval of $4.2 \pm 1.7$ years. RV performance was derived from CMR, and included RV volumes and ejection fraction (EF). LPSS was calculated globally (GLPSS) and in the RV free wall (LPSS FW), with echocardiographic speckle-tracking strainanalysis. Baseline (G)LPSS values were compared between patients and healthy controls; the relation between (G)LPSS and CMR parameters was
\end{abstract}

R. W. C. Scherptong · S. A. Mollema .

H. W. Vliegen - E. E. van der Wall .

J. J. Bax $(\bowtie) \cdot$ E. R. Holman

Department of Cardiology C5-P, Leiden University

Medical Centre, PO Box 9600, 2300 RC Leiden,

The Netherlands

e-mail: J.J.Bax@lumc.nl

R. W. C. Scherptong

e-mail: r.w.c.scherptong@lumc.nl

N. A. Blom

Department of Pediatric Cardiology, Leiden University

Medical Centre, PO Box 9600, 2300 RC Leiden,

The Netherlands

L. J. M. Kroft · A. de Roos

Department of Radiology, Leiden University Medical

Centre, PO Box 9600, 2300 RC Leiden, The Netherlands evaluated and the changes in (G)LPSS and CMR parameters during follow-up were compared. GLPSS and LPSS FW were significantly reduced in patients as compared to controls $(-14.9 \pm 0.7 \%$ vs. $-21.6 \pm$ $0.9 \%$ and $-15.5 \pm 0.9 \%$ vs. $-22.7 \pm 1.5 \%, P<$ 0.01). Moderate agreement between LPSS and CMR parameters was observed. RV EF remained unchanged during follow-up, whereas GLPSS and LPSS FW demonstrated a significant reduction. RVEF showed a $1 \%$ increase, whereas GLPSS decreased by $14 \%$, and LPSS FW by $27 \%$. RV LPSS is reduced in TOF patients as compared to controls; during follow-up RV EF remained unchanged whereas LPSS decreased suggesting that RV LPSS may be a sensitive marker to detect early deterioration in RV performance.

Keywords Tetralogy of Fallot - Echocardiography · Magnetic resonance imaging $\cdot$ Right ventricular function $\cdot$ Right ventricular strain

\section{Introduction}

Reliable quantification of right ventricular (RV) performance is essential during follow-up of patients who underwent total repair for tetralogy of Fallot (TOF) [1]. Currently, RV performance is mostly quantified with cardiovascular magnetic resonance (CMR) by assessing ventricular volumes and ejection fraction [2]. 
Although CMR provides a good estimation of $\mathrm{RV}$ performance, recent studies demonstrated that the relation with patient outcome is suboptimal [3]. This observation underscores the need for more sensitive parameters to adequately identify patients at risk for a decrease in RV performance. With the introduction of tissue Doppler imaging, the possibility emerged to evaluate RV myocardial function in patients with TOF using RV strain analysis [4]. However, tissue Doppler imaging has limitations that hamper the application of RV strain analysis in clinical practice [5]. Recently, novel echocardiographic strain analysis packages, based on speckle-tracking, have become available. This technology provides direct information on contractile performance of the right ventricle [6], which may potentially provide a more sensitive measurement to detect decreases in RV function at an early stage in patients with TOF.

The aim of the current study was twofold. First, we evaluated whether RV peak systolic longitudinal strain, quantified with 2D speckle-tracking imaging, is reduced in adult patients with TOF as compared to normal individuals. Second, we hypothesized that reduction of RV peak systolic strain during follow-up can be used for early detection of a decrease in RV performance.

\section{Methods}

Study population and protocol

The patient population consisted of 18 adult patients who underwent total correction for TOF during childhood. In adulthood, these patients were regularly followed up at the outpatient clinic for adult congenital heart disease during a period of $4.2 \pm 1.7$ years. In this study period, medical therapy was unchanged, no surgical interventions were performed and NYHA class remained stable in all patients. Characteristics are summarized in Table 1.

At the age of $33.9 \pm 11.2$ years, these patients underwent routine evaluation with echocardiography (echo) and CMR within a 3-month timeframe (baseline measurement), followed by a recent echo and CMR at the age of $38.1 \pm 11.2$ years (follow-up measurement). The echo evaluation included a standard 2D echo and 2D speckle-tracking strain analysis to calculate RV global longitudinal peak systolic
Table 1 Patient Population

\begin{tabular}{ll}
\hline & $\begin{array}{l}\text { Tetralogy of Fallot } \\
(n=18)\end{array}$ \\
\hline Male/female $(n)$ & $8 / 10$ \\
Age (years) & $33.9 \pm 11.2$ \\
BSA $\left(\mathrm{m}^{2}\right)$ & $1.8 \pm 0.24$ \\
NYHA I/II/III/IV $(n)$ & $13 / 5 /-/-$ \\
Type of repair $(n)$ & \\
$\quad$ Transannular patch & 12 \\
$\quad$ Right ventricular patch & 5 \\
$\quad$ No patch & 1 \\
Previous pulmonary valve replacement $(n)$ & 7 \\
\hline
\end{tabular}

strain (GLPSS) and longitudinal peak systolic strain separately in the RV free wall (LPSS FW). From the CMR images, RV performance was obtained, expressed as RV end systolic volume (RV ESV), $\mathrm{RV}$ end diastolic volume (RV EDV) and RV ejection fraction (RV EF).

First, we compared the LPSS values from the baseline echocardiograms of the TOF patients to that of 18 healthy controls matched for age and gender. Second, the relations between RV performance (derived from CMR) on the one hand, and GLPSS and LPSS FW (derived from echocardiography) on the other hand, were calculated. Third, we evaluated the changes in GLPSS, LPSS FW (echocardiographyderived; baseline vs. $4.2 \pm 1.7$ years follow-up) and RV performance (CMR-derived; baseline vs. $4.2 \pm$ 1.7 years follow-up). Finally, we compared the echocardiographic changes during follow-up to the changes measured with CMR.

\section{Echocardiography}

Echocardiographic imaging took place in the left lateral decubitus position on a commercially available system (Vivid Seven, General Electric-Vingmed, Milwaukee, Wisconsin). Besides the standard parasternal (long- and short-axis) and apical (2- and 4-chamber) images, additional apical (4-chamber) images were obtained that included the interventricular septum, the apex and the RV free wall up to tricuspid annulus. Images were digitally stored in the cine-loop format for off-line analysis.

Longitudinal strain was assessed off-line, on the 4-chamber cine-loop that included the RV free wall, using speckle-tracking analysis. The speckle-tracking 
software (EchoPac 7.0.0 GE Medical systems, Horten, Norway) uses natural acoustic markers, or speckles, to determine frame-to-frame movement of myocardium. Images are subdivided into blocks of approximately 20-40 pixels that contain stable patterns of speckles which are followed through the cardiac cycle [7]. In these blocks, the location of speckles is tracked with a dedicated algorithm that uses the sum of absolute differences and specific correlation criteria. After local tissue velocity vectors are derived from the spatial and temporal data of each speckle, strain can be assessed from temporal differences in the mutual distance of neighbouring speckles. For longitudinal strain assessment, the software calculates the length of a predefined segment as a percentage of the original length, shortening of a segment is represented as negative strain and lengthening of a segment is represented as positive strain.

Right ventricular longitudinal peak systolic strain was calculated in a user interface by delineating the endocardial border from the basal septum to the apex, along the RV free wall to the tricuspid valve annulus. After a region of interest was then set covering the complete width of the myocardium, the software automatically distinguished three predefined septal segments and three RV free wall segments in which longitudinal peak systolic strain was calculated. From these segments, GLPSS was obtained, which is a factory specified weighted average of all six segments [8]. Furthermore, RV LPSS FW was calculated as the average of the three free wall segments (see Fig. 1). The minimum frame rate used to calculate longitudinal strain was 40 frames per second. Two patients, in whom the image quality was considered inadequate, were excluded from further analysis.

\section{Cardiovascular magnetic resonance}

Cardiovascular magnetic resonance examinations were performed on a $1.5 \mathrm{~T}$ ACS-NT15 magnetic resonance scanner (Philips Medical Systems, Best, the Netherlands.) The scanning protocol has been described previously $[9,10]$. All images were obtained during breath holds, with an ECG triggered gradient echoplanar technique. For calculation of ventricular volumes, 10-12 consecutive slices were
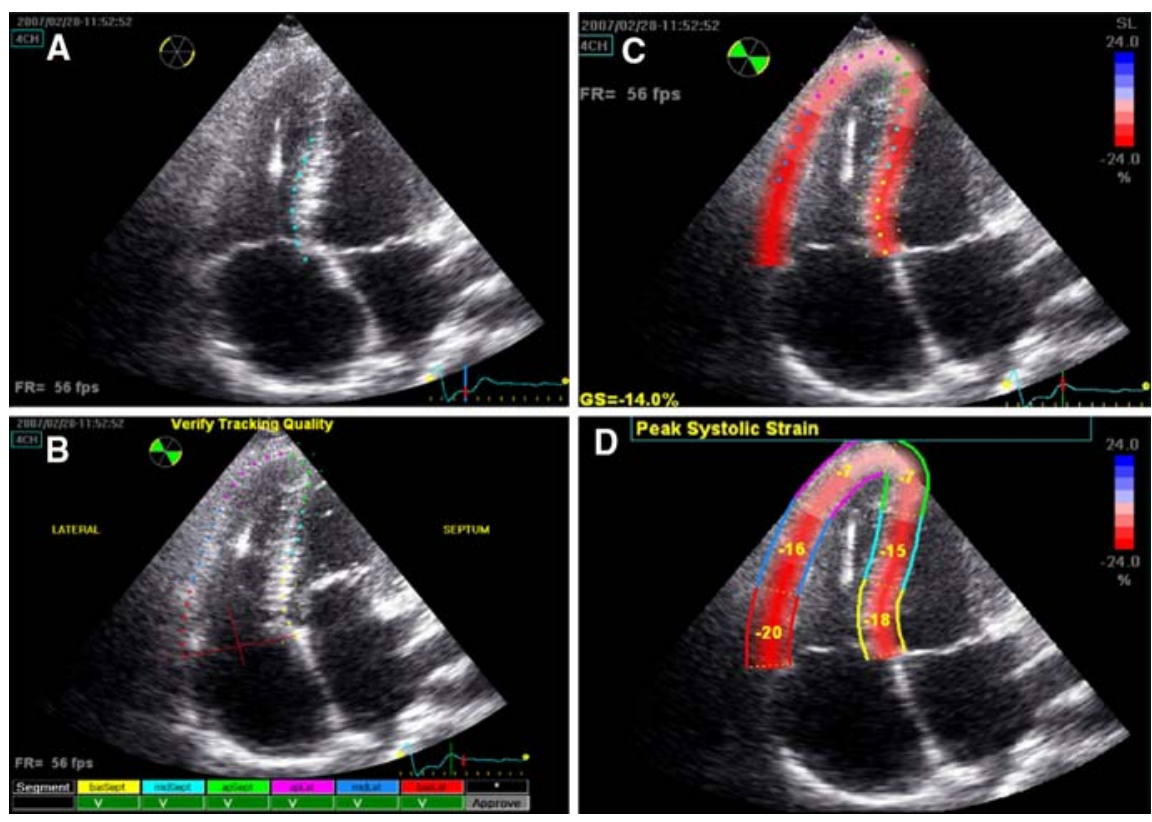

Fig. 1 2D Speckle-tracking Strain Analysis of the Right Ventricle. Right ventricular longitudinal strain analysis in the designated user interface. Panel A demonstrates how first the endocardial border is delineated in the apical 4-chamber view. Based on this contour, the interventricular septum and the right ventricular free wall are tracked automatically. The user

optimizes the tracking quality, by adjusting the aforementioned contour and by altering the region of interest (ROI), which corresponds to the width of the tracking (Panel B). Thereafter, the program calculates global strain $(G S)$ based on a weighted average of three septal and three right ventricular free wall segments (Panel $C$ and $D$ ) 
obtained in the transverse plane, to cover both ventricles from apex to base. The MASS software package (Medis, Leiden, the Netherlands), was used for calculation RV EDV, RV ESV and RV EF [11].

Statistical analysis

SPSS SPSS $^{\circledR}$ 12.0.1 for Windows, SPSS Inc., Chicago, USA) was used for statistical analysis. Data are presented as mean $\pm \mathrm{SD}$, unless mentioned otherwise. To evaluate the reproducibility of RV LPSS, the echocardiograms of 20 subjects (10 Fallot patients and 10 controls) were analyzed twice by the same observer in a random order. In addition, these echocardiograms were also analyzed by a second observer. Subsequently, intra- and interobserver agreement of GLPSS and LPSS FW was evaluated using Bland-Altman analysis. An unpaired $t$-test was used to calculate the difference between patients and healthy controls. Pearson correlation coefficients were computed to describe the correlation between echocardiography derived RV peak systolic strain and CMR derived RV function. Furthermore, the changes from baseline to follow-up were calculated using paired $t$-test analysis. $P$ values $<0.05$ were considered statistically significant.

\section{Results}

RV longitudinal peak systolic strain: patients vs. healthy controls

Table 2 demonstrates the differences in LPSS of the RV between patients with TOF and healthy controls per segment. LPSS values in patients with TOF ranged from $-12.3 \pm 7.6 \%$ in the apical septum to $-17.6 \pm 7.1 \%$ in the RV basal free wall. In healthy controls, peak systolic strain was also lowest in the apical septum $(-18.2 \pm 8.3 \%)$, but highest in the RV mid free wall $(-24.2 \pm 10.0 \%)$. The observed difference in RV LPSS between patients with TOF and healthy controls was significant in all RV segments except the apical free wall (Table 2). Furthermore, the combined strain values GLPSS and LPSS FW were significantly lower in patients with TOF as compared to healthy controls.

The intra- and interobserver agreement for both GLPSS and LPSS FW were good. The intraobserver variability was $0.4 \pm 1.4$ and $0.1 \pm 1.2$ (mean \pm 2SD), respectively, whereas the interobserver variability was $0.5 \pm 2.7$ and $0.3 \pm 1.8$ (mean $\pm 2 \mathrm{SD}$ ).

Relation between RV performance and RV longitudinal peak systolic strain in TOF patients

In Table 3, the relation between RV performance (as derived from CMR) and RV LPSS (as derived from echo) in the TOF patients is summarized. At baseline, moderate-good relations between RV volumes (as derived from CMR) and RV LPSS were observed. RV EDV correlated significantly with both GLPSS and LPSS FW, indicating reduced strain in patients with a dilated RV. RV ESV was not correlated with RV peak systolic strain values. RV EF was correlated with GLPSS and LPSS FW. At follow-up, similar correlations were observed for RV volumes and RV $\mathrm{EF}$ on the one hand, and strain values on the other hand (Table 3).
Table 2 Peak systolic strain values in tetralogy of Fallot patients vs. healthy controls

Data are presented as mean $\pm \mathrm{SE}$

$F W$ free wall, GLPSS global longitudinal peak systolic strain, LPSS longitudinal peak systolic strain, $R V$ right ventricle

\begin{tabular}{cccc}
\hline & TOF $(n=18)$ & Controls $(n=18)$ & $P$-value \\
\hline RV LPSS (segmental) & & & \\
Basal septum (\%) & $-13.1 \pm 1.1$ & $-19.6 \pm 0.7$ & $<0.001$ \\
Mid septum (\%) & $-14.4 \pm 1.0$ & $-20.2 \pm 0.7$ & $<0.001$ \\
Apical septum (\%) & $-12.3 \pm 1.8$ & $-18.2 \pm 1.9$ & 0.03 \\
Apical free wall (\%) & $-13.2 \pm 2.0$ & $-20.1 \pm 2.9$ & 0.06 \\
Mid free wall (\%) & $-15.8 \pm 1.0$ & $-24.2 \pm 1.6$ & $<0.001$ \\
Basal free wall (\%) & $-17.6 \pm 1.7$ & $-23.9 \pm 2.2$ & 0.03 \\
RV LPSS (combined) & & & $<0.001$ \\
GLPSS (\%) & $-14.9 \pm 0.7$ & $-21.6 \pm 0.9$ & $<0.001$ \\
LPSS FW (\%) & $-15.5 \pm 0.9$ & $-22.7 \pm 1.5$ & \\
\hline
\end{tabular}


Table 3 Correlation between RV performance (expressed as $\mathrm{RV}$ volumes and RV EF derived from CMR) and RV longitudinal peak systolic strain (derived from echocardiography)

\begin{tabular}{lrlll}
\hline & GLPSS $(r)$ & $P$-value & LPSS FW $(r)$ & $P$-value \\
\hline Baseline & & & & \\
RV ESV $\left(\mathrm{ml} / \mathrm{m}^{2}\right)$ & 0.01 & NS & 0.22 & NS \\
RV EDV $\left(\mathrm{ml} / \mathrm{m}^{2}\right)$ & 0.61 & 0.007 & 0.72 & 0.001 \\
RV EF $\left(\mathrm{ml} / \mathrm{m}^{2}\right)$ & -0.80 & $<0.001$ & -0.62 & 0.006 \\
Follow-up & & & & \\
RV ESV $\left(\mathrm{ml} / \mathrm{m}^{2}\right)$ & -0.37 & NS & -0.17 & NS \\
RV EDV $\left(\mathrm{ml} / \mathrm{m}^{2}\right)$ & 0.43 & 0.07 & 0.58 & 0.01 \\
RV EF $\left(\mathrm{ml} / \mathrm{m}^{2}\right)$ & -0.79 & $<0.001$ & -0.68 & 0.002 \\
\hline
\end{tabular}

$E D V$ end diastolic volume, $E F$ ejection fraction, $E S V$ end systolic volume, $F W$ free wall, GLPSS global longitudinal peak systolic strain, $L P S S$ longitudinal peak systolic strain, $R V$ right ventricle

Table 4 Changes in RV performance and longitudinal peak systolic strain over $4.2 \pm 1.7$ years follow-up

\begin{tabular}{lrrr}
\hline & \multicolumn{1}{l}{ Baseline } & \multicolumn{1}{l}{ Follow-up } & $P$-value \\
\hline RV performance & & & \\
RV ESV $\left(\mathrm{ml} / \mathrm{m}^{2}\right)$ & $49.6 \pm 14.5$ & $56.5 \pm 16.5$ & 0.020 \\
RV EDV $\left(\mathrm{ml} / \mathrm{m}^{2}\right)$ & $118.7 \pm 37.8$ & $132.5 \pm 35.5$ & 0.007 \\
RV EF (\%) & $56.8 \pm 9.3$ & $56.4 \pm 10.0$ & 0.782 \\
RV LPSS & & & \\
LPSS FW (\%) & $-15.5 \pm 3.6$ & $-12.0 \pm 5.5$ & 0.004 \\
GLPSS (\%) & $-14.9 \pm 2.8$ & $-12.9 \pm 3.7$ & 0.001 \\
\hline
\end{tabular}

$E D V$ end diastolic volume, $E F$ ejection fraction, $E S V$ end systolic volume, $F W$ free wall, GLPSS global longitudinal peak systolic strain, $L P S S$ longitudinal peak systolic strain, $R V$ right ventricle
Changes in RV performance and RV longitudinal peak systolic strain during follow-up

In Table 4, the changes in RV performance and RV LPSS are shown (baseline vs. $4.2 \pm 1.7$ years follow-up). First, whereas RV EF remained unchanged, RV EDV and RV ESV demonstrated a small, statistically significant increase, indicating that the $\mathrm{RV}$ in TOF patients exhibited gradual dilatation. Second, we observed a significant reduction in RV LPSS on both a global level (GLPSS) as well as in the RV free wall (LPSS FW). An example of a patient with significant reduction in GLPSS and LPSS FW is shown in Fig. 2. To compare the changes in RV performance and changes in RV LPSS, the overall percentual changes from baseline to follow-up were calculated and depicted in Fig. 3. RV EDV and RV ESV, respectively, demonstrated a 16 and $17 \%$ increase, whereas RV EF increased with $1 \%$. GLPSS decreased $14 \%$, the largest changes during follow-up, however, were noted in LPSS FW $(-27 \%)$.

\section{Discussion}

The main findings in the present study were that RV LPSS is reduced in patients with TOF as compared to healthy controls. Furthermore, RV LPSS was correlated with RV performance. Finally, over the follow-

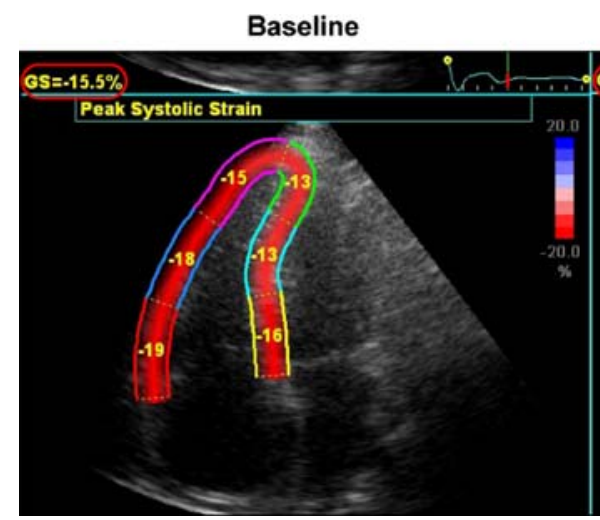

Fig. 2 Patient Example of Changes in RV Longitudinal Peak Systolic Strain in Relation to RV Performance on Cardiovascular Magnetic Resonance Imaging. In this patient example, RV end diastolic volume increased gradually from 112 to $120 \mathrm{ml} / \mathrm{m}^{2}$ over a follow-up period of 3 years, but RV ejection fraction remained unchanged $(55 \%$ at baseline vs. $54 \%$ at follow-up). Global RV longitudinal peak systolic strain,
Follow-Up

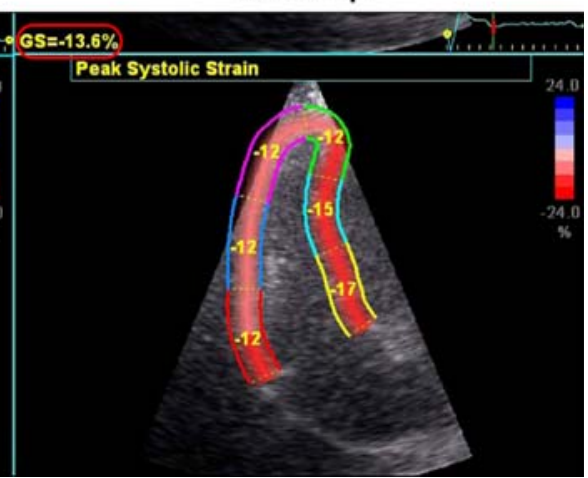

however, decreased from -15.5 to $-13.6 \%$ (red circles), mainly caused by a sharp decrease in longitudinal peak systolic strain of the RV free wall $(-17.3 \%$ at baseline vs. $-12 \%$ at 3 years follow-up). These findings indicate that RV performance measured with CMR may exhibit only minimal changes (without change in RV EF), whereas RV LPSS already decreases 


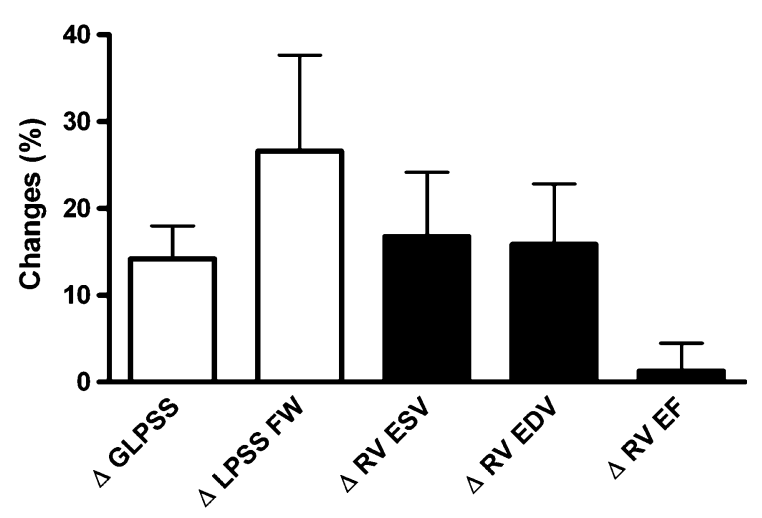

Fig. 3 Changes in Right Ventricular Performance and Right Ventricular Longitudinal Peak Systolic Strain. The white bars represent the overall changes in RV longitudinal peak systolic strain whereas the black bars represent the overall changes in cardiovascular magnetic resonance derived RV volumes and ejection fraction. To compare the relative magnitudes in changes between strain and volumes/ejection fraction, the percentual changes from baseline to follow-up (rather than the absolute changes) are shown. A significant change was noted for RV volumes, global longitudinal peak systolic strain (GLPSS) and longitudinal peak systolic strain in the RV free wall (LPSS FW). The largest changes were observed in LPSS FW

up period, a significant reduction in RV LPSS was observed, both on a global level (GLPSS) and in the RV free wall (LPSS FW), accompanied by a slight RV dilatation, but in the absence of changes in RV EF.

After total repair for TOF, a gradual deterioration of RV performance is observed in most patients [1], which is associated with a gradual reduction of functional class and the need for (surgical) interventions [3]. Accordingly, early detection of a decrease in $\mathrm{RV}$ performance is important in the routine followup of patients with TOF.

Generally, CMR is the preferred technique for quantification of RV performance, and the most traditional parameters include RV volumes and RV EF [12]. Various CMR studies have been performed to evaluate the relation between RV performance and outcome in patients with TOF $[9,10,13]$. Although these studies demonstrated that a severely dilated RV with depressed RV EF $(<35 \%)$ was associated with poor functional performance (NYHA class III) and outcome, accurate prediction of adverse outcome based on CMR derived RV performance measurements is limited [3, 14]. Moreover, exercise capacity appeared a strong predictor of adverse outcome in patients with TOF [15], but CMR derived RV performance measurements were demonstrated to be poorly related with exercise capacity [13]. Thus, there appears a need for more sensitive parameters of $\mathrm{RV}$ performance to allow for earlier detection of a decline in RV performance. In the current study, we demonstrated that echocardiography derived RV strain measurements may provide this information. It was shown that RV LPSS was reduced in patients with TOF as compared to healthy individuals; in TOF patients, GLPSS was $-14.9 \pm 0.7 \%$, as compared to $-21.6 \pm 0.9 \%$ in healthy controls. Although literature data on RV LPSS in adult patients with TOF are lacking, previous studies in children after total repair for TOF also reported reduced RV strain values [4, 16]. In a study by Weideman et al, RV LPSS was calculated with tissue Doppler imaging in pediatric TOF patients and compared to healthy controls [4]. The authors reported RV LPSS of $-20 \%$ in both the interventricular septum as well as in the RV free wall. In a similar study by Solarz et al. [16], in which strain also was calculated with tissue Doppler imaging, LPSS values of $-17 \%$ in the RV free wall and of $-21 \%$ in the interventricular septum were described. RV LPSS was more reduced in the current study $(-14.9 \pm 0.7 \%$ in TOF patients vs. $-21.6 \pm 0.9 \%$ in normal controls), which may have been related to differences in patients age between study populations in the present vs. former studies. The mean age of patients in the current study was $34 \pm 11$ years, whereas the mean age in the previous studies was $11 \pm 3$ years and $7 \pm 4$ years, respectively [4, 16] Furthermore, both the timing as well as the type of total correction is most likely different in the current study population as compared to previously described pediatric populations. As a consequence of the evolution of the surgical approach to TOF, the earlier timing of total correction over the years and the use of smaller transannular patches, younger patients are less prone to the development of $\mathrm{RV}$ dysfunction [17]. In addition, difference in strain calculation technique, tissue Doppler imaging vs. speckle-tracking may provide an alternative explanation for the differences between the current study and former studies.

The relation between RV LPSS and GLPSS derived from 2D speckle-tracking on the one hand, and RV performance derived from CMR parameters on the other hand was evaluated in the current study. We observed moderate-good correlations between 
RV LPSS with echocardiography and RV performance assessed by CMR, both at baseline and at follow-up. Patients with severe RV dilatation demonstrated reduced GLPSS and LPSS FW as compared to patients with preserved RV dimensions (Table 3). Furthermore, patients with depressed RV EF had lower GLPSS and LPSS FW as compared to patients with preserved RV EF (Table 3). The relation between the CMR derived RV performance measurements, and the echocardiography derived strain measurements was not excellent, suggesting that the two parameters provide different information.

This suggestion is further strengthened by the observation that despite unchanged RV EF on CMR over the follow-up period (RV EF $56.8 \pm 9.3 \%$ at baseline vs. $56.4 \pm 10.0 \%$ at follow-up, $P=\mathrm{NS}$ ), the RV strain parameters exhibited already some deterioration over time (Table 4; Fig. 3), suggesting that strain parameters may provide more sensitive information on RV performance as compared to the traditional $\mathrm{RV} \mathrm{EF}$ and $\mathrm{RV}$ volumes derived from CMR.

To the best of our knowledge, this is the first study to demonstrate the course of RV strain deterioration in patients with TOF. One of the pathophysiological substrates, besides the presence of RV dilatation, underlying this subtle deterioration of RV performance is likely to be related to gradual formation of myocardial fibrosis [18]. Babu-Narayan et al. [19] recently reported that patients with corrected TOF develop fibrosis in the RV as visualized on contrastenhanced CMR. In the patient population of 92 adult TOF patients aged $32 \pm 11$ years, the authors demonstrated that RV fibrosis was present in $99 \%$ of the patients after 4-41 years follow-up. In this study, the presence of fibrosis was not only related to RV dilatation, but also to the increased incidence of arrhythmias and reduced exercise capacity, indicating that RV fibrosis formation is an important mechanism in patients with TOF [19]. Possibly, the presence of RV fibrosis, also explains the reduced RV LPSS values in our current population.

\section{Limitations}

As a shortcoming of most studies in patients with congenital heart disease, the sample size of the current study was small. Therefore, the relation of RV GLPSS and LPSS FW to pulmonary regurgitation, which is an important determinant of RV function in TOF, could not be investigated. Furthermore, the current observations need to be related to outcome, in order to demonstrate whether the changes in RV LPSS without changes in RV EF are of clinical relevance.

\section{Conclusion}

The current findings showed that RV longitudinal peak systolic strain is reduced in TOF patients as compared to normal controls. Moreover, RV longitudinal peak systolic strain correlates moderately with measures of RV performance on CMR. Although RV ejection fraction on CMR remained unchanged and only modest RV dilatation was present during follow-up, echo-derived longitudinal peak systolic strain measures already decreased, suggesting that longitudinal peak systolic strain may be more sensitive to detect changes in RV performance. Further studies are needed to evaluate the role of RV longitudinal peak systolic strain to detect abnormalities in RV performance at an early stage.

Open Access This article is distributed under the terms of the Creative Commons Attribution Noncommercial License which permits any noncommercial use, distribution, and reproduction in any medium, provided the original author(s) and source are credited.

\section{References}

1. Atik FA, Atik E, da Cunha CR et al (2004) Long-term results of correction of tetralogy of Fallot in adulthood. Eur J Cardiothorac Surg 25:250-255

2. van Straten A, Vliegen HW, Hazekamp MG et al (2005) Right ventricular function late after total repair of tetralogy of Fallot. Eur Radiol 15:702-707

3. Geva T, Sandweiss BM, Gauvreau K et al (2004) Factors associated with impaired clinical status in long-term survivors of tetralogy of Fallot repair evaluated by magnetic resonance imaging. J Am Coll Cardiol 43:1068-1074

4. Weidemann F, Eyskens B, Mertens L et al (2002) Quantification of regional right and left ventricular function by ultrasonic strain rate and strain indexes after surgical repair of tetralogy of Fallot. Am J Cardiol 90:133-138

5. Gondi S, Dokainish H (2007) Right ventricular tissue Doppler and strain imaging: ready for clinical use? Echocardiography 24:522-532

6. Perk G, Tunick PA, Kronzon I (2007) Non-Doppler twodimensional strain imaging by echocardiography-from technical considerations to clinical applications. J Am Soc Echocardiogr 20:234-243 
7. Leitman M, Lysyansky P, Sidenko S et al (2004) Twodimensional strain-a novel software for real-time quantitative echocardiographic assessment of myocardial function. J Am Soc Echocardiogr 17:1021-1029

8. Reisner SA, Lysyansky P, Agmon Y et al (2004) Global longitudinal strain: a novel index of left ventricular systolic function. J Am Soc Echocardiogr 17:630-633

9. Vliegen HW, van Straten A, de Roos A et al (2002) Magnetic resonance imaging to assess the hemodynamic effects of pulmonary valve replacement in adults late after repair of tetralogy of fallot. Circulation 106:1703-1707

10. Oosterhof T, van Straten A, Vliegen HW et al (2007) Preoperative thresholds for pulmonary valve replacement in patients with corrected tetralogy of Fallot using cardiovascular magnetic resonance. Circulation 116:545-551

11. Niezen RA, Helbing WA, van der Wall EE et al (1996) Biventricular systolic function and mass studied with MR imaging in children with pulmonary regurgitation after repair for tetralogy of Fallot. Radiology 201:135-140

12. Rebergen SA, Chin JG, Ottenkamp J et al (1993) Pulmonary regurgitation in the late postoperative follow-up of tetralogy of Fallot. Volumetric quantitation by nuclear magnetic resonance velocity mapping. Circulation $88: 2257-2266$

13. Meadows J, Powell AJ, Geva T et al (2007) Cardiac magnetic resonance imaging correlates of exercise capacity in patients with surgically repaired tetralogy of Fallot. Am J Cardiol 100:1446-1450

14. Knauth AL, Gauvreau K, Powell AJ et al (2008) Ventricular size and function assessed by cardiac MRI predict major adverse clinical outcomes late after tetralogy of Fallot repair. Heart 94:211-216

15. Giardini A, Specchia S, Tacy TA et al (2007) Usefulness of cardiopulmonary exercise to predict long-term prognosis in adults with repaired tetralogy of Fallot. Am J Cardiol 99:1462-1467

16. Solarz DE, Witt SA, Glascock BJ et al (2004) Right ventricular strain rate and strain analysis in patients with repaired tetralogy of Fallot: possible interventricular septal compensation. J Am Soc Echocardiogr 17:338-344

17. Giannopoulos NM, Chatzis AC, Bobos DP et al (2004) Tetralogy of Fallot: influence of right ventricular outflow tract reconstruction on late outcome. Int J Cardiol 97(Supp1 1):87-90

18. Oosterhof T, Mulder BJ, Vliegen HW et al (2005) Corrected tetralogy of Fallot: delayed enhancement in right ventricular outflow tract. Radiology 237:868-871

19. Babu-Narayan SV, Kilner PJ, Li W et al (2006) Ventricular fibrosis suggested by cardiovascular magnetic resonance in adults with repaired tetralogy of fallot and its relationship to adverse markers of clinical outcome. Circulation 113:405-413 